\title{
Nuclear Transport at Low Excitations
}

\author{
Helmut Hofmann ${ }^{1}$ and Fedor A.Ivanyuk ${ }^{2}$ \\ 1) Physik-Department der TU München, D-85747 Garching, Germany* \\ 2) Institute for Nuclear Research, 252028 Kiev-28, Ukraine ${ }^{\dagger}$
}

June 23, 2018

\begin{abstract}
Numerical computations of transport coefficients at low temperatures are presented for shapes typically encountered in nuclear fission. The influence of quantum effects of the nucleonic degrees of freedom is examined, with pair correlations included. Consequences for global collective motion are studied for the case of the decay rate. The range of temperatures is specified above which this motion may be described as a quantal diffusion process.
\end{abstract}

PACS numbers: 05.60.Gg, 24.10.Pa, 24.75.+i, 25.70.Ji

Phys. Rev. Lett. 82 (1999) 4603

In the past decade much progress has been made in the understanding of nuclear transport phenomena in the regime of not too low temperatures, say between 1 and $5 \mathrm{MeV}$ (with $k_{B}=1$ ). Such a situation is reached if two heavy ions collide at an energy above the Coulomb barrier, but where the excess energy per particle still is small compared to the Fermi energy. In this regime the dynamics of the composite system may be parameterized in terms of shape variables. Of particular interest is the outgoing channel which is dominated by fission and the emission of light particles and $\gamma$ 's. It has been possible experimentally to deduce valid information on the time scale of collective motion [1], and, hence, on the size of nuclear dissipation. These experiments suggest collective motion to be over-damped, possibly providing an answer to the question raised by Kramers as early as 1940 in his seminal paper [2], namely whether nuclear friction is "abnormally small or abnormally large". Nowadays such processes are described theoretically in terms of the Langevin equation [3], which is understood to be equivalent to Kramers' original equation (of Fokker Planck type) for the density in collective phase space.

\footnotetext{
*e-mail:hhofmann@physik.tu-muenchen.de
}

†e-mail: ivanyuk@kinr.kiev.ua 
On general grounds, it may be anticipated that the magnitude of nuclear dissipation will vary with excitation. Indeed, there are experimental indications [4] for such a conjecture. At small thermal excitations the dynamics is governed by the (real) mean field for which there is no room for damping of slow collective motion like fission. At larger $\mathrm{T}$ coupling to more complicated configurations sets in, which causes transfer of energy from the collective degrees of freedom $Q_{\mu}$ to the nucleonic ones $x_{i}$. Within the linear response approach [5] the effects of this coupling are accounted for by dressing the single particle energies by complex self-energies depending both on frequency and $\mathrm{T}$. Approximating its imaginary part by a constant proportional to $T^{2}$, friction will again decrease with $T$, once the microscopic damping becomes so large that one may speak of "collision dominance". At intermediate temperatures there might be the intricate contribution to friction from the "heat pole", which has been seen to be large for nonergodic systems [6], and which has a dramatic influence on the T-dependence.

In the present Letter we want to focus on very low excitations, say in the range below $T \approx 1 \mathrm{MeV}$. This regime not only is of great practical importance, as for the production of super-heavy elements [7], for instance, it is also of great theoretical interest. First of all, there is little doubt that in this domain quantum effects dominate nucleonic dynamics, and the transport coefficients will strongly be influenced by shell effects and pair correlations. One even must expect quantum features to be present for collective motion, for instance as corrections to Kramers formula for the decay rate [8]. Often quantal approaches are based on the functional integral method applied to simplified Hamiltonians of the Caldeira-Leggett type (for a review see [9]). There, the bath degrees of freedom $x_{i}$ are represented by a set of oscillators of fixed frequencies, with a bilinear coupling between the $x_{i}$ and the collective variable $Q$. The decay rate is calculated for imaginary time propagation. Both features hardly can be taken over to nuclear fission. First of all, the simplest constraint to warrant self-consistency between the mean field and the shape of the nuclear density requires the former to change with $Q$. This aspect alone makes it very difficult to work with a (pre-fixed) Hamiltonian for the total system of all degrees of freedom. Moreover, the temperature, which one may define [10] for the fast degrees of freedom (supposedly be given by the "nucleonic" ones), is subject to changes with $Q$ as well as with time. The latter feature occurs because of the evaporation of particles mentioned before.

For these reasons a formulation with real time propagation is much more appropriate. This has been achieved by a suitable application of linear response theory on the basis of a locally harmonic approximation (LHA) (for a review see[5]). One exploits the concept of propagators which move the system forward in collective phase space by small time steps. As the individual ones only cover small areas they may be represented by (multidimensional) Gaussians. The latter satisfy an equation of motion whose structure is similar to that of Kramers, with only the diffusive terms being modified to account for quantum effects.

The following study is based on numerical calculations of transport coefficients for average motion, namely friction $\gamma$, inertia $M$ and local stiffness $C$, more precisely of 
those ratios which determine transport in phase space,

$$
\Gamma_{\gamma}=\frac{\gamma}{M}, \quad \varpi^{2}=\frac{|C|}{M}, \quad \eta=\frac{\Gamma_{\gamma}}{2 \varpi}=\frac{\gamma}{2 \sqrt{M|C|}}
$$

Their knowledge will allow us to examine implications on the diffusion coefficients and, hence, on transport processes like fission. It would be most desirable that such information be used as input for computational codes to solve for Fokker-Planck or Langevin equations. In this way one would be able to examine in more detail the role of shell effects, which are known to produce structure not only in the static energy but in the inertial and frictional forces as well. To simplify matters, for the present purpose we will look at the more schematic case where the system's energy exhibits just one minimum and one barrier at $Q_{a}$ and $Q_{b}$, respectively. The stiffnesses and the barrier height are found from a Strutinsky calculation of the free energy. Finer details of shell effects are removed both from the potential as well as from the transport coefficients by applying a suitable smoothing over a small region of the collective variable around $Q_{a}$ and $Q_{b}$.

Suppose we may at first discard any quantum effects in the collective degrees of freedom, which amounts to look at the "high temperature limit", for which Kramers' equation applies [5]. The temperatures we have in mind are always small compared to the barrier height, $T \ll E_{b}$. The decay rate $R_{K}$ then shows the following behavior. For given $T$, but as function of the $\eta_{b}$ (at the barrier), the $R_{K}\left(\eta_{b}, T\right.$ ) increases first, to decrease after it has reached a maximal value (see e.g. [11]). The decreasing branch is represented well by Kramers' "high viscosity limit" [2]

$$
R_{K}=\frac{\omega_{a}}{2 \pi}\left(\sqrt{1+\eta_{b}^{2}}-\eta_{b}\right) \exp \left(-E_{b} / T\right)
$$

which is valid for $\eta_{b} \geq T /\left(2 E_{b}\right)$ (see e.g. [9]). If blindly extended down to $\eta_{b}=0$ this form $R_{K}$ reaches a value typical for a simple transition state model, $r_{T S T} \approx r\left(\eta_{b}=0\right.$ ) (BohrWheeler formula). Rather, for very small $\eta_{b}$, one ought to apply Kramers' "low viscosity limit", given by $R_{K}^{l . v .}=\Gamma_{\gamma}^{b}\left(E_{b} / T\right) \exp \left(-E_{b} / T\right)$. For nuclear physics the latter has not played any role yet, as $\eta$ is believed to lie above the limit given below (2). According to [5, 13, 12 this should be the case at temperatures above $1 \mathrm{MeV}$. Moreover, the $\varpi$ does not change much with $T$. In [12] a value of about $1 \mathrm{MeV} / \hbar$ was found both at the potential minimum as well as at the saddle. It so turns out that this feature is more or less recovered even at smaller $T$, say within an accuracy of the order of $20 \%$, which may be good enough for the following discussion.

More drastic modifications are expected, and indeed seen, for dissipation. To study this behavior, the $\gamma, \Gamma_{\gamma}$ and $\eta$ have been calculated on the basis of the same deformed shell model as in [12 but with pair correlations included. The transformation from independent particles to quasi-particles of BCS-type is standard. For our purpose the common procedure does not suffice, however. As mentioned previously, a decent and sensible description of nuclear dissipation needs to account for "collisions". At low thermal excitations their effects, too, will strongly be influenced by pair correlations. Look at the extreme case of zero temperature and take an even-even system for the sake of simplicity. Then there will be no quasi-particle states within twice the gap energy 
$2 \Delta$. Hence, the imaginary part of the self-energy $\Gamma(\hbar \omega, \Delta, T=0)$ must be zero at least within such a range of frequencies $\hbar \omega$. Hence, at $T=0$ friction will strictly vanish for any collective motion whose frequency $\omega=\varpi$ lies in that range, i.e. $\hbar \omega \leq 2 \Delta$. Extrapolating from the case of $T=0$ to finite $T$ we should expect the function $\gamma=\gamma(T)$ to have a step like behavior. This dependence then goes through to that of $\Gamma_{\gamma}$ and $\eta$, albeit the inertia, too, is influenced by pairing.

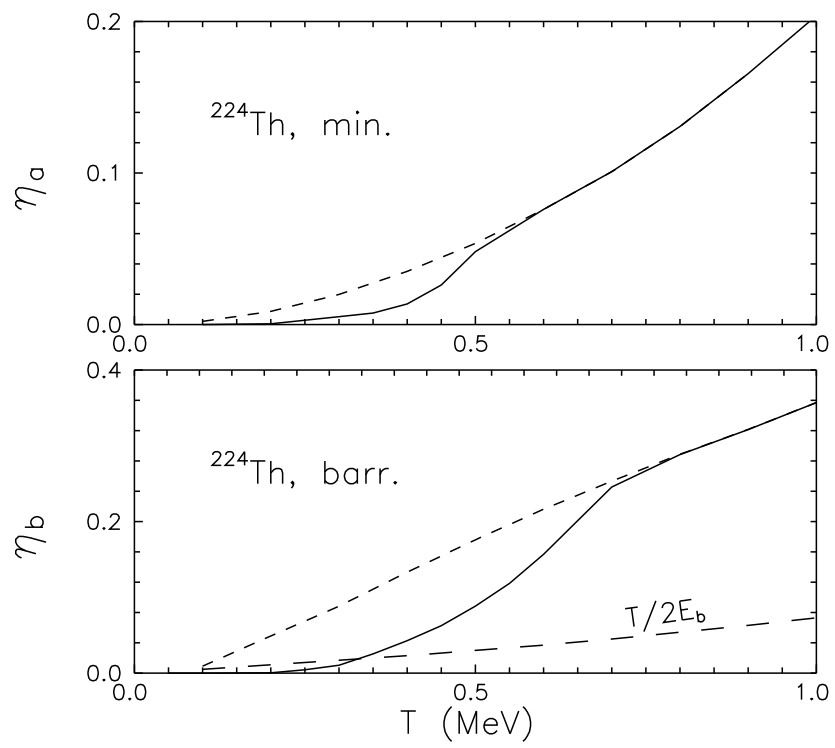

Figure 1: The damping factor $\eta=\gamma /(2 \sqrt{M|C|})$ at the potential minimum (top) and at the fission barrier (bottom) of ${ }^{224} T h$; the dashed curves are for $\Delta=0$. The long-dashed line in the bottom part shows the function $T / 2 E_{b}$.

Let us demonstrate these features on the basis of numerical calculations performed for the example of ${ }^{224} \mathrm{Th}$. For details we have to refer to [14], but we may mention that the $\Gamma(\hbar \omega, \Delta, T)$ has been calculated along the lines suggested in [15]. In Fig.1 we display the $\eta(T)$ 's at the minimum and the barrier. They have been obtained for a $\Delta=\Delta(T)$ as determined by the gap equation. Unfortunately, so far it has not been possible to calculate the underlying response function $\chi(\omega)$ in full glory. Rather, when evaluating the necessary folding integrals over frequency the correct width had to be approximated by a constant, calculated at the Fermi energy $\mu: \Gamma(\hbar \omega, \Delta, T)=\Gamma(\hbar \omega=\mu, \Delta, T)$. Indeed, in this regime of small $\omega$ and for $T \leq T_{\text {pair }}$ where pair correlations disappear, such an estimate may be considered to represent the correct width well enough to allow for a general analysis. Evidently, the values of $\eta$ 's obtained for $\Delta \neq 0$ clearly fall well below those of the unpaired case, shown here by the dashed lines.

The most important features exhibited in Fig.1 may be summarized as follows, together with the consequences for Kramers' decay rate: (1) The step like function mentioned before is clearly visible. (2) Below $T \approx T_{\text {pair }} \simeq 0.5 \mathrm{MeV}$ the effective damping rate $\eta$ is smaller than about 0.1. (3) As seen in Fig.1, $\eta$ may fall below $T / 2 E_{b}$ such that formula (2) no longer applies. (4) Up to $T \simeq 1 \mathrm{MeV} \eta$ stays below $\simeq 0.2$ at the minimum and below $\simeq 0.3$ at the barrier. The latter value implies that the rate may be 
approximated fairly well by the transition state value $r_{T S T} \approx R_{K}(\eta=0)$, see (2). (5) These values of $\eta$ are much smaller than those one gets within "macroscopic models", say in terms of a combination of wall friction with the stiffness and inertia of the liquid drop model (with irrotational flow).

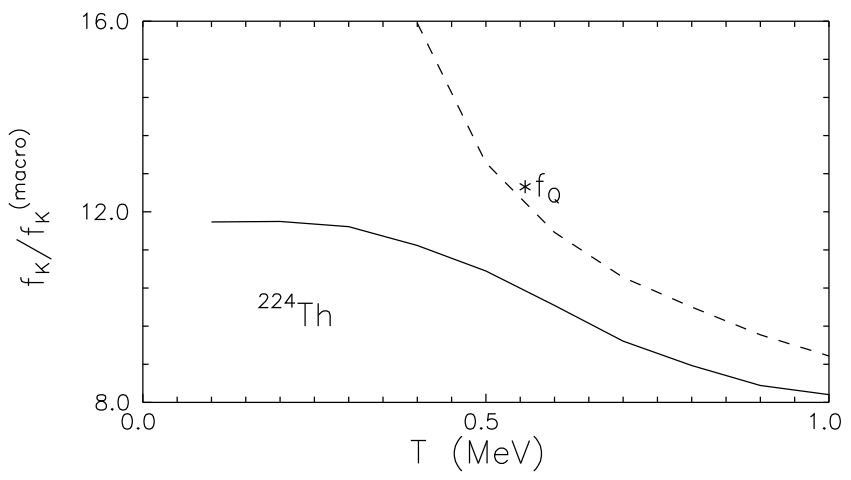

Figure 2: The ratio of the Kramers correction factor $f_{K}=\sqrt{1+\eta_{b}^{2}}-\eta_{b}$ to its macroscopic counterpart as function of temperature. The quantity $f_{Q} f_{K} / f_{k}^{\text {macro }}$ accounts for the quantum correction factor $f_{Q}$, see eq.(5).

In Fig. 2 we plot the ratio of Kramers' pre-factor $f_{K}=\left(\sqrt{1+\eta_{b}^{2}}-\eta_{b}\right)$ obtained from our results to that of the macroscopic limit just described [16]. It is seen that the latter underestimates the decay rate by about a factor of 10. Quantum corrections will increase this deviation further, indicated here by the dashed curve.

Within the LHA, these quantum corrections come in through the diffusion coefficients, as given by the fluctuation dissipation theorem [5]. It is only at temperatures above $2 \mathrm{MeV}$ that one may safely assume the classic Einstein relation $D_{p p}=\gamma T$ to be valid [5]. In the general case, in addition to the $D_{p p}$ there is a cross term $D_{q p}$, both of which depend in non-linear way on combinations of $M, \gamma$ and $C$, or on the parameters introduced in (1). The diffusion coefficients behave very differently for stable and for unstable modes. To demonstrate this feature let us look at the limit of small dissipation $\eta \ll 1$. To lowest order in $\gamma$ one gets

$$
D_{q p}=0 D_{p p}=\gamma T^{*} \text { with } T^{*}(\varpi)=\frac{\hbar \varpi}{2} \operatorname{coth}\left(\frac{\hbar \varpi}{2 T}\right)
$$

with $\varpi=\sqrt{C / M}=|\varpi|$ for $C>0$ and $\varpi=i|\varpi|$ for $C<0$. The form (3) may be said to represent the correct behavior fairly well below $\eta \simeq 0.1$ (see Fig.3.4.2 of [5]). From the results shown above for $\eta(T)$, one may thus argue the relation (3) to be acceptable for temperatures below $T_{\text {pair }}$, whereas deviations must be expected for $T \geq T_{\text {pair }}$. For $C<0$ and weak friction the diffusion coefficient $D_{p p}$ falls below the values given by the Einstein relation. It quickly drops to zero at a critical temperature $T_{c}$, below which the $D_{p p}$ would become negative and the diffusion equation would loose its meaning. The value of this $T_{c}$ decreases with increasing $\eta$, such that the form given in (3) delivers an upper limit and we may write

$$
T_{c} \leq T_{c}(\eta=0)=\hbar \varpi_{b} / \pi \quad T_{c}<T_{\text {pair }}
$$


The statement on the right is reached assuming the $\hbar \varpi_{b}$ to be of the order of $1 \mathrm{MeV}$ and taking the value for $T_{\text {pair }}$, as reported above, together with the fact that below $T_{\text {pair }}$ the damping rate $\eta$ falls below 0.1 .

Commonly, the quantum corrections to Kramers decay rate are expressed by a factor $f_{Q}$ appearing in the correct rate as $R=f_{Q} R_{K}$ (see e.g. [9]). As shown in [8], this form may also be obtained within the LHA. This derivation is based on the assumption that in the neighborhood of the potential minimum friction is large enough to ensure sufficient relaxation inside the well. The same assumption is behind Kramers' "high viscosity limit", upon which we just have convinced ourselves to be given in the range of temperature at $T_{\text {pair }}$ and above. Moreover, this assumption turns out to be necessary also when the problem is formulated and solved with path integrals in real time propagation (see [17]).

The $f_{Q}$ can be expressed by a ratio of two partition sums: $f_{Q}=\left|\mathcal{Z}_{b}\right| / \mathcal{Z}_{a}$, where the one associated to the barrier has to be defined by analytic continuation. According to [18], the $\mathcal{Z}$ of a damped oscillator can be calculated from the equilibrium fluctuations of momentum and coordinate. Hence, within the LHA it might be expressed by the diffusion coefficients. Unfortunately, for $\gamma \neq 0$ a calculation of the momentum fluctuation requires regularization, for instance by introducing a frequency dependent friction coefficient (Drude regularization). To get a fairly simple estimate of $f_{Q}$ and its T-dependence we used the following formula (with $\hbar \omega_{n}=n 2 \pi T$ )

$$
f_{Q}=\prod_{n=1}^{\infty} \frac{\omega_{n}^{2}+\omega_{n} \overline{\Gamma_{\gamma}}+\varpi_{a}^{2}}{\omega_{n}^{2}+\omega_{n} \overline{\Gamma_{\gamma}}-\varpi_{b}^{2}} \quad \text { with } \quad \overline{\Gamma_{\gamma}}=\frac{\Gamma_{\gamma}^{a}+\Gamma_{\gamma}^{b}}{2}
$$

It may be noted in passing, (a) that without (Drude) regularization this formula would diverge for $\Gamma_{\gamma}^{a} \neq \Gamma_{\gamma}^{b}$ and (b) that problems of this type are absent for the Caldeira-Leggett approach where the transport coefficients do not change with the collective variable; generalizations are possible, though, for instance by introducing variable coefficients phenomenologically, see e.g. [19]. The result of a numerical evaluation of (5) within our theory is shown in Fig.2 by the dashed curve. This graph demonstrates several features, valid in this range of temperatures: (i) The quantum effects in the collective motion may change the decay rate by about $30 \%$ or less. (ii) Already at $T=1 \mathrm{MeV}$ they only amount to about $10 \%$. (iii) More important are the quantum effects of nucleonic motion, which are responsible for the deviation of the transport coefficients from the macroscopic models.

Unfortunately, it is not possible to carry the analysis further down to smaller temperatures. Below $T_{c}$ [recall (田] we have seen the LHA break down. It may be said that this problem generally appears in real time formulations. Within the functional integral approach this has been demonstrated in [17] and traced back to the harmonic approximation to the barrier. The problem at stake here is a very severe one for any applications of Langevin or Fokker Planck equations to nuclear physics. Both methods allow one to account for various non-linear effects, as manifested by variable transport coefficients, for instance, but both of them rely on "real time propagation". Moreover, practical computer programs exploit locally harmonic approximations, in one way or other, such that it is not possible to even define meaningful diffusion coefficients below $T_{c}$. 
In this context we should like to mention the possibility of calculating and exploiting a Feynman-Vernon functional for global motion on the basis of Random Matrix Theory [20]. In principle, this might allow one to study quantum effects, but it is somewhat questionable whether this procedure will be applicable to nuclear physics, at those temperatures where these quantum effects become important which we just mentioned. This concern has essentially two reasons - discarding for the moment the very fact that this model, too, has difficulties with self-consistency. (i) Generally an application of RMT ceases to be valid at low excitations. (ii) So far practical applications have been possible only to leading order in an expansion in $1 / T$, actually to that regime of $T$ where friction decreases with $T$.

Summarizing our results we hope to have been able to exhibit for low energy nuclear physics an exciting problem of quantum transport which still is lacking a general solution. With respect to the application in nuclear physics itself, it appears to be very difficult to describe theoretically processes like the "cold" production of super heavy elements without a decent understanding of transport at small temperatures and weak dissipation. Whereas low thermal excitations are dictated by experimental conditions, the fact of small friction then is a consequence of the quantal nature of nucleonic dynamics in a mean field, in particular when pair correlations become important. Of course, to obtain more quantitative results, further studies on the microscopic level are needed. For instance, it is necessary to understand better the mechanism of "collisions" under the presence of pair correlations. Their role on the T-dependence of transport, as well as that of the "heat pole" in the larger range of excitations, require further clarification. Likewise, it should be very interesting to allow for fluctuations in the gap parameter and to examine in which way they might modify fission dynamics.

The authors like to acknowledge fruitful discussions with J. Ankerhold and N.V. Antonenko. This work was supported by the Deutsche Forschungsgemeinschaft.

\section{References}

[1] D. Hilscher, I.I. Gontchar and H. Rossner, Physics of Atomic Nuclei 57, 1187 (1994); P. Paul and M. Thoennessen, Ann. Rev. Part. Nucl. Sci. 44, 65 (1994); J.P. Lestone, Phys. Rev. Lett. 70, 2245 (1993) and Tours Symposium on Nuclear Physics II, Tour, France, (World Scientific, Singapore, 1995), p.458, ISBN 981-02-2156.

[2] H.A. Kramers, Physica 7, 284 (1940).

[3] K.Pomorski, J.Bartel, J.Richert and K. Dietrich, Nucl. Phys. A605, 87 (1996); P.Fröbrich and I.I.Gontchar, Phys. Rep. 292, 131 (1998); Y. Abe, S. Ayik, P.G. Reinhard, E.Suraud, Phys. Rep. 275, 49 (1996).

[4] D.J.Hofman, B.B.Back and P.Paul, Phys. Rev. C 51, 2597 (1995); J. Wilczyński, K. Siwek-Wilczyńska and H.W. Wilschut, Phys. Rev. C 54, 325 (1996); G. Rudolf, talk presented at the RIKEN-Symposium, March, 13-14, 1998 (to be published).

[5] H. Hofmann, Phys. Rep. 284, 137 (1997). 
[6] H. Hofmann, F.A. Ivanyuk and S. Yamaji, Nucl. Phys. A598, 187 (1996).

[7] For the "cold" mechanism see: P. Armbruster, GSI-Preprint-98-96; see also G.G.Adamian, N.V.Antonenko, W.Scheid and V.V.Volkov, Nucl.Phys. A633, 409 (1998); for "warm" fusion see: Yu.A.Lazarev, Yu.V.Lobanov, Yu.Ts.Oganessian et al, Phys. Rev. C 54, 620 (1996).

[8] H. Hofmann, G.-L. Ingold and M. Thoma, Phys. Lett. B 317, 489 (1993).

[9] P. Hänggi, P. Talkner and M. Borkovec, Rev. Mod. Phys. 62, 251 (1990).

[10] To simplify matters we want to discard here the problem of possible fluctuations of $T$, which may be deduced from the formula $\Delta T / T=1 / \sqrt{\mathcal{C}}$. Estimating the specific heat $\mathcal{C}$ from the Fermi gas model one obtains $\Delta T / T=1 / \sqrt{A T}(\mathrm{~T}$ in $\mathrm{MeV})$.

[11] F. Scheuter and H. Hofmann, Nucl. Phys. A394, 477 (1983).

[12] F.A. Ivanyuk, H. Hofmann, V.V. Pashkevich and S. Yamaji, Phys. Rev. C 55, 1730 (1997); nucl-th/9701032.

[13] S. Yamaji, F.A. Ivanyuk and H. Hofmann, Nucl. Phys. A612, 1 (1997).

[14] F.A. Ivanyuk and H. Hofmann (to be published)

[15] P.J. Siemens and A.S. Jensen, Single particle dynamics in: eds. R. Broglia et al. (Elsevier Sience Publishers B.V., Amsterdam, 1986).

[16] These macroscopic models are related to the high temperature limit of our microscopic approach, see e.g. [5].

[17] G.-L. Ingold, Thesis (PHD), Univ. Stuttgart 1988; J. Ankerhold, H. Grabert and G.-L. Ingold, Phys. Rev. E 51, 4267 (1995); J. Ankerhold and H. Grabert, Phys. Rev. E 52, 4704 (1995), ibid E 55, 1355 (1997).

[18] H. Grabert, U. Weiss and P. Talkner, Z. Physik B 55, 87 (1984).

[19] P. Fröbrich and G.-R. Tillack, Nucl. Phys. A540, 353 (1992).

[20] A. Bulgac, G. Do Dang and D. Kusnezov, Phys. Rev. E 58, 196 (1998). 